\title{
La religión en la ciencia contemporánea: impertinencias e inspiración*
}

\author{
SANTIAGO COLLADO GONZÁLEZ \\ Grupo de Investigación “Ciencia, Razón y Fe” (CRYF) \\ Universidad de Navarra, Pamplona \\ scollado@unav.es
}

Resumen. Este trabajo ofrece una panorámica de las diversas posturas que se han dado respecto a las relaciones que mantienen la ciencia y la religión. Se pone de manifiesto la dificultad de clasificarlas y la insuficiencia de las propuestas taxonómicas más comunes. Se propone ver dicha relación desde la perspectiva de las impertinencias cometidas de una respecto de la otra y, también, desde los beneficios que mutuamente se han aportado. Desde este enfoque se vislumbra un cuadro más complejo y rico que el que se puede ofrecer desde una mera clasificación. También se descubre que, cuando desde uno de estos ámbitos se rechaza o desprecia lo que corresponde al otro, se acaba usurpando su papel y asumiéndolo como propio, pero de una manera pobre o completamente desenfocada. La consecuencia no puede ser otra que el escepticismo.

Palabras clave: ciencia y religión; epistemología; fe e ideologías.

Abstract. Religion in contemporary science: impertinence and inspiration. The present article provides an overview of the different positions throughout history on the connections between science and religion. We expose the difficulty to classify these connections and the deficiency in the taxonomic proposals. This study is intended to revise this interplay from the perspective of the impertinences committed to each other, as well as from the benefits that one has received from the other. From this point of view, we can get a glimpse of a more complex and rich scene than that offered

La primera versión de este artículo ha aparecido en: Muñoz, R., J. Sánchez-Cañizares, and G. Guitián, eds. 2012. Religión, Sociedad Moderna y Razón Práctica. Pamplona: EUNSA.

ScientiaetFides 1(1)/2013, 63-85 
by a simple classification. We also reveal that, when one of these fields ignores what pertains to the other and finally rejects it, it ends up either taking its role or accepting it as its own, but in a poor or completely unfocussed way. The consequence of this is unavoidably scepticism.

Keywords: science and religion; epistemology; faith and ideologies.

\section{Vigencia del debate ciencia-religión}

El título de este artículo nos coloca ante un debate que se ha reeditado de distintas formas y con diversos nombres a lo largo de la historia. La presencia de la religión en la ciencia contemporánea alude a un debate que, según mi opinión, se encuadra en otro más amplio y antiguo y que podríamos denominar como debate fe y razón. La ciencia es un tipo particular de racionalidad y da unos tonos particulares a la relación entre la fe y la razón que son característicos de nuestro tiempo. Aunque, lógicamente, se mantienen cuestiones de fondo que son comunes a todas las formas que ha adoptado dicha relación.

La vigencia en nuestros días del debate es obvia. Basta con recordar, por ejemplo, el reciente juicio de Dover, en el estado de Pensilvania, al finalizar el año 2004 y dar comienzo el 2005. La sentencia prohibió leer en las clases de ciencias de los colegios estatales un texto crítico con la teoría de la evolución. El manifiesto dejaba abierta la puerta, en opinión de muchos, a una supuesta alternativa a la teoría evolutiva actual: el llamado "Diseño Inteligente” (DI). La sentencia venía a afirmar que la lectura de dicho texto, como había prescrito el equipo directivo del colegio demandado, era enseñar religión en la clase de ciencias y no propiamente ciencia. El juez determinó que el Diseño Inteligente era religión y no ciencia. No deja de ser llamativo que sea un juez quien tenga que determinar lo que es religión y lo que es ciencia.

Más reciente y más cercano es el caso de la suspensión, hace unos cuatro años, de algunas conferencias ya anunciadas en algunas universidades públicas españolas que iban a ser impartidas por defensores del citado movimiento DI. La razón aducida fue evitar polémicas. Es también muy 
reciente el debate, más mediático que científico, que suscitó la publicación de un nuevo libro del carismático astrofísico Stephen Hawking titulado "El Gran Diseño". La mayor intensidad del debate se produjo, curiosamente, antes de que el libro alcanzara las librerías. De hace pocos meses es la polémica provocada por el manifiesto de un grupo de científicos, encabezados por Richard Dawkins, en el que se solicitaba al gobierno la prohibición de la enseñanza del creacionismo y del "Diseño Inteligente" en los colegios públicos ingleses. El mismo Richard Dawkins mantuvo más tarde un debate público con el entonces primado de la Iglesia Anglicana Rowan Williams, al que titularon "Human Beings \& Ultimate Origin”. El debate fue seguido con gran expectación y concurrencia en el "Sheldonian Theatre" de Oxford y tiene ya miles de consultas en YouTube a pesar de haber tenido lugar tan sólo el pasado 23 de febrero.

\section{Contexto del debate}

El debate ciencia-religión se convierte en tema explícito de reflexión en el ámbito académico especialmente en el siglo XIX. Lo cual implica una nueva objetivación de los términos que entran en el debate en ese momento. Para gran parte de los históricos de la ciencia actuales, la ciencia, tal como hoy la entendemos, comienza a ejercerse precisamente en el siglo XIX. También se ha defendido más frecuentemente que nace en los siglos XVI-XVII. Pienso que se pueden hacer compatibles ambas posiciones ya que, lo que tiene lugar en el renacimiento es el inicio y la configuración del método experimental. Dicho método constituye el núcleo del modo en que las ciencias empíricas son ejercidas en nuestros días.

Si se admite que la noción de ciencia frente a la de filosofía natural se formula en el siglo XIX, no es extraño que sea también entonces cuando comienzan a aparecer los primeros estudios académicos sobre la relación entre la ciencia y la religión. Pero dichos estudios se realizan sobre la base de las objetivaciones entonces vigentes de ambas “disciplinas". El paradigma de disciplina científica de entonces era la Física. En el siglo XIX la Física no había sufrido todavía el terremoto que, en el cambio de siglo, zarandeó 
los fundamentos del gran edificio de la mecánica newtoniana. Los éxitos cosechados hasta entonces por la física clásica, y el nacimiento de la nueva biología en la segunda mitad del siglo XIX parecían disolver todo límite de la ciencia en su pretensión de explicar la realidad.

La ciencia, como empresa racional, se comenzó a comprender en el XIX según un tipo particular de objetivación que fue consolidándose a lo largo de ese siglo. También es hoy asumido por la mayoría de los históricos que, para que se pudiera elaborar un discurso sobre la relación ciencia-religión, era necesaria una objetivación de la religión que tuviera contornos bien definidos y que dicha objetivación se formó sólo unos pocos años antes. Peter Harrison, por ejemplo, director del "Ian Ramsey Centre for Science and Religion", defiende que la noción de ciencia nace en el XIX, y la noción de religión que se confronta con ella es formulada unos decenios antes, durante el desarrollo de la ilustración europea, en la estela de la fragmentación posterior a la Reforma (Harrison 2010, 31). Según este autor, se trata de una noción de religión objetivada como el conjunto de afirmaciones o creencias a las que se presta asentimiento. Por tanto, se pierde de vista o se resta importancia al compromiso vital, en todas sus dimensiones, que comporta la práctica religiosa. En definitiva se trata de una visión parcial de la religión, impuesta por las exigencias académicas y el tipo de racionalidad entonces imperante, en la que se enfatizan los elementos racionales en detrimento del resto. Una ventaja de este tipo de compresión de la religión consistía, entonces y ahora, en que ofrecía la posibilidad de abordar también estudios comparativos entre distintas religiones y no sólo entre ellas y la ciencia.

Estas simplificaciones tuvieron, y todavía tienen, gran incidencia en el modo de abordar el debate entre ciencia y religión. No pocas veces, la consecuencia ha sido plantear el debate de una manera reductiva. Dicha reducción ha llevado, especialmente en el siglo XIX, a interpretaciones estereotipadas y dependientes de diversas orientaciones ideológicas.

Por una parte es problemático hablar de ciencia en singular. La pretensión de una teoría del todo que permita "explicar", con un conjunto limitado de leyes, la totalidad de la realidad material, es el sueño del pro- 
yecto reduccionista. Pero la realidad se muestra tozuda, y nos obliga a enfrentarnos con ella desde una pluralidad de ciencias irreductibles entre sí.

No todas las ciencias tienen el mismo nivel de madurez, de formalización y de incidencia en las cuestiones filosóficas o teológicas. Es además muy problemático asumir una noción de ciencia que no se vea obligada a tener en cuenta las diferencias profundas existentes entre las diversas disciplinas, y que pueda considerarse como algo aislado e independiente del resto de los saberes. Si esto fuera posible, la ciencia se relacionaría con otros ámbitos del saber no científicos de una manera externa. Efectivamente se simplificaría el estudio de las relaciones tipo ciencia-religión, pero el precio sería ofrecer un cuadro de la religión, de la ciencia y de sus relaciones claramente insuficiente. En nuestros días esa imagen parece estar ya siendo superada y, consiguientemente, somos más conscientes de la complejidad que ofrecen este tipo de estudios. El hecho es que el debate ciencia-religión sigue estando vigente quizás más que nunca, y que los estudios que versan sobre él han recibido en los últimos años un fuerte impulso.

Otra muestra muy reciente del creciente interés por este tema, también para la Iglesia Católica, es el anuncio de la constitución por Benedicto XVI, a petición del cardenal Gianfranco Ravasi, de la fundación „Ciencia y Fe”. Es la primera de este tipo que se crea en la Santa Sede.

Una causa que sin duda ha impulsado los estudios de la relación entre ciencia y religión en nuestros días ha sido el apoyo económico que la Fundación Templeton ha ofrecido para la realización de este tipo de estudios durante los últimos decenios.

\section{Categorización}

Si se quiere aportar un poco de luz sobre el tema que nos ocupa, debemos buscar una vía para escapar a los estereotipos todavía frecuentes en nuestros días. Si no hay ciencia sino ciencias y si, además, es preciso tener en cuenta las diferencias entre las diversas religiones, parece claro que nuestro objetivo se complica enormemente. Tratar de hacer una exposición 
del papel de la religión en la ciencia contemporánea, como dice el título, escaparía completamente del alcance de una exposición como ésta.

Algunos estudiosos de la historia y la filosofía de la ciencia han tratado de poner orden en el debate, y han estableciendo un conjunto de categorías en las cuales poder incluir los distintos tipos de relaciones propuestas por los académicos. La dificultad que presenta la diversidad con la que cabe entender el significado de ambos términos, se ha tratado de superar centrando la atención en una religión determinada o en una ciencia particular. Especialmente en los estudios del siglo XIX, la religión que se ha considerado como modelo de referencia ha sido la religión cristiana. Es principalmente a ésta a la que se refieren las consideraciones que siguen.

Agustín Udías (2010, 85-129), por ejemplo, propone una clasificación de la relación entre ciencia y religión basada en la formulada originariamente por Ian Barbour. La de Udías contiene cinco categorías, una más que la de Barbour: conflicto, independencia, diálogo, complementariedad e integración. Estas clasificaciones sirven para sistematizar un ámbito temático que, como hemos dicho, resulta muy complejo. Exponemos a continuación los rasgos resumidos de las categorías mencionadas.

La tesis del conflicto irrumpe inicialmente y está presente con fuerza, sobre todo, en estudios de finales del siglo XIX. Los autores más famosos que defienden esta tesis son Draper y White. Aunque Draper es más radical en su visión negativa respecto al papel desempeñado por la Iglesia que White, ambos se apoyan en el caso Galileo como paradigma del conflicto permanente entre ambas. Los títulos de sus trabajos más representativos en este ámbito son una expresión clara de las tesis que sostienen: "Historia del conflicto entre ciencia y religión” (1875) de White y, "Una historia de la guerra de la ciencia con la teología en el Cristianismo" (1896) de Draper. Los autores que sostienen esta tesis ven en la religión y, en particular en el catolicismo, un obstáculo para el desarrollo de la ciencia. Su relación con ella siempre será de conflicto por "la prioridad que se otorga a la fe frente a la razón”. Hoy en día hay autores como Dawking o Dennet que siguen manteniendo esta tesis. Defender esta posición implica una simplificación extrema de las relaciones entre ciencia y religión. 
La tesis de la independencia entre los dos ámbitos es la vía que algunos proponen para defender la inexistencia de conflicto entre ambos. Cuando dicho conflicto se produce, es por no entender que ciencia y religión se ocupan de ámbitos que son completamente independientes. No hay intersección entre ellos y, consiguientemente, no hay realmente conflicto. El conocido biólogo y prolífico escritor Stephen Jay Gould, fallecido hace diez años, acuñó la noción de "non overlapping magisteria" para denominar esta posición que él también defendía. Con diferentes matices, el biólogo español Francisco Ayala, premio Templeton del año 2010, ha defendido en sus publicaciones tesis que son equivalentes y que, a mi juicio, se pueden incluir dentro de esta categoría.

La tesis del diálogo defiende la necesidad de mantener una relación constante entre los dos ámbitos precisamente para evitar el conflicto. La apertura de una "disciplina" hacia la otra es el único camino que permite disipar los posibles conflictos que surjan entre ambas. El diálogo libra a la ciencia de ideologías y permite hacer una teología que tenga en cuenta los conocimientos que la ciencia está aportando continuamente sobre la realidad.

La tesis de la complementariedad, la mantenida por ejemplo por autores como Hans Küng, defiende que cualquier tipo de conocimiento, incluido el religioso, nos ofrece una visión parcial de la realidad. Son necesarias, por tanto, las aportaciones de todos los ámbitos del saber para poder formarnos una visión comprensiva de la realidad. Lo que en realidad se mantiene en esta postura es una separación entre ciencia y religión semejante, desde el punto de vista gnoseológico, a la que sostienen los defensores de la tesis de la independencia. Lo peculiar en este caso es que se ponen en un plano de igualdad metódico el conocimiento aportado por las dos esferas estudiadas. La posición que se defiende es semejante a un perspectivismo que hace necesarios, y también equivalentes epistemológicamente, los dos saberes. La complementariedad sería una consecuencia de conseguir sumar saberes diversos más que de integrarlos realmente, como propone la categoría siguiente.

La tesis de la integración es la mantenida, por ejemplo, por Pierre Teilhard de Chardin. Yo también incluiría ahí, aunque sus propuestas sean muy 
distintas a las de Teilhard, al reciente movimiento denominado "Diseño Inteligente". Los situados en esta categoría defienden, en general, que la ciencia permite ir confirmando los conocimientos aportados por la fe o, también, que la fe nos ofrece verdades que son útiles y productivas para la ciencia. En esta categoría encontramos, por tanto, las posiciones que defienden de una manera más radical la unidad entre ciencia y fe. Algunos de los exponentes de esta posición son tan radicales que, en realidad, hacen desaparecer, o diluyen en gran medida lo que otros han denominado autonomía metodológica de la ciencia y la religión. Es fácil advertir que esta última categoría de propuestas se puede considerar como un "cajón de sastre" donde incluir todas las que no encajan bien en alguna de las anteriores. Aquí también se podría incluir, por tanto, la posición de la Iglesia Católica. El problema es que el "cajón de sastre" es demasiado grande y se guardan en él perspectivas que incluso entran en conflicto entre sí.

Es patente que esta clasificación, aun siendo útil, plantea numerosos problemas. No parece estar formulada con un criterio unívoco. Por ejemplo, las dos primeras categorías obedecen a un criterio que podríamos calificar de compatibilidad: la primera afirma la incompatibilidad entre ciencia y religión y la segunda la perfecta compatibilidad. No obstante la razón por la que la segunda categoría defiende la compatibilidad podría dar lugar a otro criterio de clasificación de carácter epistemológico. En cambio, la tercera categoría, la del diálogo, pone el énfasis en una disposición deseable en los actores implicados y explica la existencia o no del conflicto sobre la base de la consecución o no de dicha actitud dialogante. Se podría decir que el criterio empleado en este caso es de carácter sociológico. La tesis de la complementariedad parece razonable aunque, como ya he señalado, la considero insuficiente y, me parece, aborda el problema de una manera epistemológicamente desenfocada. En realidad no da cuenta de las diferencias de carácter metódico que hay entre ambos ámbitos. Ya hemos señalado también algunos de los problemas que presenta la última categoría.

Si atendemos exclusivamente al criterio de la compatibilidad entre la ciencia y la religión, me parece menos problemático reducir las posibles categorías de relación entre la ciencia y la religión a cuatro: conflicto, in- 
dependencia, integración y mutua colaboración o armonía (Carlson 2000). Entiendo en este caso las dos primeras y la integración como en la clasificación anterior. Pero en lugar de incluir en la clasificación las categorías del diálogo y de la complementariedad, me parece más adecuado eliminarlas y hablar de una nueva que califico de mutua colaboración o armonía.

En este caso el criterio de clasificación es más uniforme y tiene que ver con la compatibilidad entre ciencia y religión, incluyendo en cada categoría grupos de propuestas más afines desde el punto de vista epistemológico. De esta manera se puede evitar incluir en la misma categoría a propuestas que son epistemológicamente diferentes como, por ejemplo, "Diseño Inteligente”, Theilard y la Iglesia Católica. En particular, considero que a la Iglesia Católica se debe incluir dentro de la categoría mutua colaboración y armonía. La Iglesia Católica defiende la autonomía metodológica de la ciencia y la religión sin renunciar a su mutua colaboración y armonía (Gaudium et Spes, nn. 35-36; Fides et Ratio, prólogo). Dicha posición mantiene un equilibrio sutil entre autonomía y armonía que se sustenta, a mi juicio, gracias a que se afirma la existencia de niveles autónomos de racionalidad que interaccionan entre sí de una manera diversa según los niveles considerados.

No obstante, esta segunda clasificación mantiene algunos de los problemas señalados para la primera. Estimo que sería ingenuo pensar que las complejas relaciones entre la ciencia y la religión, o mejor, entre las religiones y las diversas ciencias, pueden encerrarse, sin matices, en alguna de ellas. Si nos referimos al cristianismo en general, la complejidad de las interacciones nos obliga a admitir que las relaciones entre religión y ciencia han pasado por todas y cada una de dichas categorías a lo largo del tiempo y el espacio. Incluso se puede apreciar la coexistencia de diferentes tipos de relaciones en un mismo tiempo. Los historiadores se refieren a esta situación proponiendo lo que denominan como "tesis de la complejidad". Del mismo modo que ocurre con cualquier otro ámbito o tema, una mera clasificación siempre es insuficiente para dar cuenta de la realidad que se estudia.

No obstante, el subtítulo de esta exposición, nos sugiere cómo ordenar nuestro tema según un criterio diferente. Buscamos examinar la relación 
entre ciencia y religión, renunciando a la imposible aspiración de ser exhaustivos, pero sin faltar al rigor. Examinaremos las relaciones entre los dos polos del debate desde otro punto de vista. Se puede decir que a lo largo de la historia ha habido fructíferas inspiraciones y lamentables impertinencias en ambos sentidos. Y, por el tiempo disponible, me limitaré a algunas de las que considero más importantes.

Examinaremos en primer lugar algunas de las que podrían considerarse fuentes de inspiración tanto de la ciencia para la religión como de la religión para la ciencia. A continuación abordaremos el problema de las impertinencias que puedan haberse dado en ambos sentidos. Finalmente, trataré de esbozar una conclusión sobre algunas causas subyacente a las impertinencias, es decir, dar cuenta de la causa de los conflictos, según las dos clasificaciones descritas al inicio.

\section{Inspiración}

\subsection{Religión como fuente de inspiración de la ciencia}

Propongo que la religión, en particular la religión cristiana, ha sido y es fuente de inspiración para la ciencia al menos en dos sentidos: en las convicciones culturales que han hecho posible el origen de la ciencia, y en los presupuestos intelectuales y morales necesarios para que la actividad científica se desarrolle.

La convicción de la racionalidad del mundo y de que el hombre, con su intelecto, es capaz de desentrañar dicha racionalidad ha sido, según autores como Stanley Jaki (Jaki 1990; 2002), André Maurois, Pierre Duhem, Pierre Chaunu, Whitehead (Whitehead 1967, 1-18) o el mismo Mariano Artigas (Artigas 1989; 1999, 310-347), una de las causas que ha hecho posible la aparición y el desarrollo de la ciencia actual. La investigación en muchos ámbitos científicos, quizá todos, se inició y se ha continuado desde la convicción de que el mundo, al ser criatura de Dios, posee una racionalidad intrínseca. Dios es su Logos, es el origen de su verdad. Podemos por tanto controlar, dominar el mundo - poner nombre a todas las cosas-, gracias 
al uso de la razón. Somos capaces de comprender y encontrar sentido en aquello que nuestro conocimiento nos presenta.

Establecer leyes que determinan el comportamiento de la realidad, encontrar patrones que la naturaleza siempre cumple por ser ésta fruto de la sabiduría eterna y bondadosa de Dios, que la naturaleza no sea un caos sin orden y sin posibilidad de ser controlada, ha sido, según los autores mencionados, una condición que ha favorecido la aparición de la ciencia. Aunque esta propuesta no ha sido pacíficamente aceptada en todo momento y, obviamente, no haya sido la única causa o fuente de inspiración, sí parece coherente que la creencia en el Dios creador cristiano haya sido un elemento catalizador e impulsor de la actividad científica.

El nacimiento de la ciencia moderna está vinculado al nacimiento de las universidades. Los principales hombres de la Edad Media que crearon un clima intelectual propicio para el nacimiento de la ciencia tuvieron que ver con el nacimiento de las universidades. Esta institución nace, como es bien sabido, por iniciativa de la Iglesia. Los que hoy son considerados principales precursores de la ciencia moderna, como Roger Bacon (1214-1294), que acuñó el nombre de ciencia experimental, Robert Grosseteste (1175-1253), Alberto Magno (1206-1280), Jean Buridan (1295-1358) o Nicolás Oresme (1320-1382), fueron hombres de fe.

Es también interesante destacar la idea propuesta por algunos autores como Duhem, según la cual, condenas como las que el obispo de París Tempier impuso a Siger de Brabante (1240-1284) y sus seguidores en los años 1270 y 1277 , habrían constituido un impulso para que muchos pensadores exploraran otras doctrinas no aristotélicas. Considera Duhem que esto fue uno de los múltiples factores que hicieron posible la aparición de la ciencia experimental (Ferngren 2002, 57-72).

Los presupuestos filosóficos de la actividad científica tienen que ver también con el sustrato cultural que, según Jaki, es necesario para el nacimiento de la ciencia. De dichos presupuestos se ha ocupado con detalle y amplitud Mariano Artigas. Están relacionados o son equivalentes a aquellos que según los autores mencionados hicieron posible o, al menos, facilitaron la aparición de la ciencia moderna. Artigas defiende que toda acti- 
vidad científica supone la existencia de un orden inteligible en la realidad física y, por otra parte, que tenemos la capacidad de conocer ese orden. La fe cristiana ha afirmado siempre la racionalidad del mundo por ser este una criatura de Dios. La fe ha servido a los creyentes dedicados a la actividad científica, o intelectual en general, para reforzar los supuestos de la actividad científica señalados por Artigas (Artigas 2000, 39-40).

\subsection{La ciencia fuente de inspiración para la religión}

Hay dos orientaciones que se pueden abordar para desarrollar este epígrafe. La primera consiste en determinar si la ciencia lleva a los científicos o a la sociedad, que reciben un influjo cada vez mayor de la ciencia, hacia una mayor religiosidad o, por el contrario, son empujadas por la ciencia hacia el ateísmo. El segundo consistiría en determinar si las aportaciones procedentes de la ciencia al conocimiento humano y, por tanto, a la cultura en general, determinan de alguna manera el modo de entender la religión o incluso modificar la misma comprensión de las verdades reveladas.

Abordar con rigor cualquiera de los dos enfoques exigiría mucho más espacio de lo que se nos permite decir aquí. Faltan además estudios sistemáticos y suficientemente amplios y "científicos" como para poder responder con un mínimo de garantías a las preguntas anteriores. Sí podemos hacer, en cambio, una breve reflexión sobre estos modos de relación de la ciencia con la religión y, simplemente, constatar que sería interesante llevar a cabo investigaciones serias sobre este particular.

Sobre si la ciencia lleva o no a la religión, cabe destacar una realidad ya mencionada anteriormente: muchos de los protagonistas del inicio y desarrollo de la ciencia, como Copérnico, Galileo, Newton, Volta, Ampère, Cauchy, Faraday, Kelvin, Maxwell, Planck, Millikan, Marconi o Lemaître, entre otros, han sido hombres de fe. Este hecho es relevante tanto si estudiamos la inspiración en un sentido como en el contrario. Al menos nos lleva a pensar que la ciencia no tiene porqué ser un obstáculo para la profesión de una fe religiosa. Ciertamente también hay científicos agnósticos y ateos. Darwin, por ejemplo, parece que fue evolucionando a lo largo 
de su vida pasando de ser una persona de fe en los años de su juventud, a abrazar un agnosticismo, de contornos poco definidos, en los años de su madurez. Lo difícil es concluir que la causa de ese proceso interior fuera la consecuencia de su investigación científica. Hay otras razones de índole personal y familiar que para algunos constituyen una razón más verosímil de su evolución religiosa.

En cambio, sí son claramente significativas de que un científico puede encontrar motivos de inspiración religiosa en el ejercicio de su ciencia, las palabras del físico italiano Carlo Rubbia (Nobel de física en el año 1984) en una entrevista publicada en el Neue Zürcher Zeitung en 1992:

Cuando enumeramos galaxias o probamos la existencia de partículas elementales, probablemente no estamos demostrando la existencia de Dios. Pero como científico y estudioso me impresionan profundamente el orden y la belleza que encuentro en el cosmos y dentro de los fenómenos materiales. Y como observador de la naturaleza no puedo rechazar la noción de que aquí hay un orden superior de cosas. Encuentro absolutamente inaceptable la idea de que todo sea el resultado de la coincidencia o una mera diversidad estadística. Aquí existe una inteligencia superior, por encima y más allá de la existencia misma del Universo.

Por otra parte, conocidos ex-ateos como Antony Flew, o agnósticos en su momento como Francis Collins, reconocen el papel que la ciencia ha tenido en sus respectivas conversiones.

Se han realizado ya algunos estudios, sobre todo de carácter estadístico y mediante encuestas, sobre el papel que la religión desempeña en la vida de prestigiosos científicos actuales.

En uno de ellos, centrado en particular en biólogos que trabajan en prestigiosas universidades de los Estados Unidos, se concluía que sólo un 10\% de los encuestados (149 biólogos expertos en evolución) veían conflicto entre la ciencia que practicaban y las creencias religiosas (Graffin and Provine 2008). La mayoría de ellos no veían ningún conflicto entre ciencia y religión. Los autores del trabajo dicen que los participantes, aunque no ven conflicto entre ambas, sin embargo no suelen creer en Dios, la existencia del espíritu u otras creencias básicas para la religión. Además también 
muchos de ellos piensan que es la ciencia la que explica la existencia de la religión, y no se considera la influencia en sentido contrario. Este es el motivo por el que la mayoría de los encuestados no ven incompatibilidad. La opinión de los que consideran que son compatibles por la existencia de un "doble magisterio" es, en este estudio, minoritaria.

Otro estudio más reciente y extenso sobre la "religiosidad" de los científicos ha sido realizado por Elaine Howard Ecklund y Elizabeth Long (Ecklund and Long 2011). En este caso, fueron preguntados 275 científicos de 21 universidades "top" en Estados Unidos. Las autoras muestran su sorpresa ante el hecho, inesperado para ellas, de que la mayoría de los científicos encuestados se consideraban a sí mismos como personas "espirituales". Ciertamente piensan que esa "espiritualidad" constituye una especie de sustituto de las religiones institucionales. También perciben que este tipo de espiritualidad parece ser distinto en algunos aspectos a la que viven personas que se dedican a otras actividades.

Resumiendo mucho, algunas de las conclusiones que las autoras extraen de su trabajo son las siguientes: se podría decir que el científico es propenso a considerarse a sí mismo como espiritual; que dicha espiritualidad es compatible en bastantes de ellos con el agnosticismo y, también pero en menor medida, con el ateísmo (hay algunos ateos que se consideran a sí mismos "espirituales”); que dicha espiritualidad supone una búsqueda de sentido a la realidad que su trabajo les pone de manifiesto. Buscan respuestas a interrogantes que les surgen en su trabajo, pero los encuestados no preguntan a las "religiones". Para ellos, las religiones tradicionales tienen un significado que es equivalente a "dogma institucionalizado". Esta visión impregna con un tinte negativo a la palabra religión, ya que es percibida como contraria a la indagación y búsqueda personal: algo que está en el núcleo de la actividad científica. Ser espiritual en lugar de religioso, en cambio, parece que sí es compatible con una búsqueda personal, lo cual parece más compatible con la práctica científica ejercida por estos.

Las autoras del trabajo concluyen que, aunque es patente un proceso de secularización en el ámbito científico, la dedicación a la investigación hace que dicho proceso tenga unas características que le son propias. El hecho 
de que los científicos entrevistados se consideren personas espirituales aunque no religiosas tiene su origen, según las autoras, en el "deseo de comprender la inmensidad y la complejidad del orden natural, el cual surge inicialmente de su ciencia y lleva a un sentimiento de asombro y maravilla que trasciende dicho orden natural" (Ecklund and Long 2011, 271).

El otro aspecto mencionado sobre si la religión misma y sus contenidos se ven afectados por el desarrollo de la ciencia es, como el anterior, muy complejo de abordar con rigor. Pienso que, efectivamente, la ciencia ejerce en cierta medida dicho influjo, pero que habría que matizar esta afirmación con detenimiento. A lo largo de la historia, los descubrimientos de la ciencia han influido en la evolución de las ideas filosóficas, incluidas aquellas que han servido para explicar el contenido de la fe cristiana. Por tanto, de una manera al menos indirecta, la ciencia influye en la misma Teología. No quiero decir con esto que haya modificado, en el caso del catolicismo, el contenido de la fe de la Iglesia. Pero sí ha servido, por ejemplo, para ayudar a separar la componente humana de la revelación, con la que el contenido revelado se expresa, del auténtico contenido de las verdades reveladas. La componente humana de la revelación está condicionada por las diversas cosmovisiones de cada época. Esta influencia ha sido especialmente clara en el ámbito de la exégesis de los textos bíblicos.

Es muy ilustrativa la lectura de algunos de los textos que el santo Juan Pablo II escribió en referencia a este tema. El siguiente está en el número 8 del discurso pronunciado el 23 de abril de 1993, con motivo del centenario de la encíclica Providentissimus Deus y el cincuentenario de la Divino afflante Spiritu:

[Se trata de] comprender el sentido de los textos con toda la exactitud y precisión posible y, por tanto, en su contexto histórico y cultural. Una falsa idea de Dios y de la Encarnación empuja a un cierto número de cristianos a seguir una orientación contraria. Estos tienen la tendencia a creer que, siendo Dios el Ser absoluto, cada una de sus palabras tiene un valor absoluto, independiente de todos los condicionamientos del lenguaje humano. [...] Cuando [Dios] se expresa en un lenguaje humano, no da a cada expresión un valor uniforme, sino que utiliza los posibles matices con extrema flexibilidad, y acepta también sus limitaciones. 
De todo lo dicho en este apartado se puede concluir que el ateísmo de los científicos no es tanto una consecuencia del ejercicio de su actividad científica y de las actitudes y hábitos que con dicha actividad se promueven, sino más bien se puede inferir que depende de los mismos procesos culturales que afectan a otros ámbitos de la sociedad y la cultura. Más aún, el científico que trabaja en las ciencias naturales descubre una racionalidad en el mundo que le invita a remontarse a un logos, a una razón originaria. Pero esto ya sería entrar en un tipo de racionalidad de carácter filosófico que supone abrir una puerta a la fe, aunque no sea una causa suficiente para tenerla, o mejor, para recibirla.

En definitiva la ciencia invita a hacerse planteamientos de tipo religioso, aunque a esto se le llame simplemente planteamientos de tipo espiritual. A lo que no parece que invite es al ateísmo. Por tanto, pienso que podría decirse que la ciencia constituye un tipo particular de inspiración para la religión y que ha ayudado a comprender mejor aspectos parciales relacionados con la revelación.

\section{Impertinencias}

Pero no todo ha sido, ni es, relación amable entre ciencia y religión. No han sido pocos los autores que han defendido tesis que se pueden considerar fuera de la categoría que hemos llamado "Mutua colaboración y armonía". Cualquier tipo de relación que pueda incluirse dentro de las tres primeras categorías considero que encierra, en mayor o menor medida, algún tipo de impertinencia por alguna de las partes. En cualquier caso hay posiciones que a mi juicio son más impertinentes que otras. Además, las impertinencias se han producido tanto contra la ciencia como contra la fe.

\subsection{Impertinencias contra la ciencia}

Comencemos, aunque seamos ya en este apartado especialmente breves, por las impertinencias que provienen de particulares posiciones religiosas. En realidad han sido ya señaladas en párrafos anteriores al establecer 
la clasificación propuesta al inicio. Concretamente nos vamos a detener en un tipo concreto. La llamaré impertinencia fundamentalista. Un caso particular de dicha impertinencia sería el creacionismo. Sostengo que ser creacionista no es precisamente defender que el mundo ha sido creado por Dios, sino defender el hecho de la creación según un tipo particular de interpretación de la Biblia literalista, que entra en conflicto con resultados científicos ampliamente contrastados: evolución de las especies, edad de la Tierra, etc.

La impertinencia procedería de tratar de imponer al científico una interpretación particular de los hechos que éste explica mediante su ciencia, desde fuera del método que los mismos científicos han desarrollado para explicarlos. La impertinencia es clara y procede del intento, normalmente bien intencionado, de defender la "fe" contra aquello que se percibe como una amenaza, porque contrasta con una particular interpretación de la palabra escrita revelada. Es esto lo que considero propiamente un fundamentalismo creacionista.

La realidad, con sus múltiples procesos y dinamismos, no es fácil de entender y controlar. Las ciencias naturales, que persiguen tanto el conocimiento verdadero de lo físico como su control, han desarrollado herramientas intelectuales de gran sofisticación para conseguirlo. Algunas de las consecuencias que el uso de dichas herramientas comporta, son un verdadero desafío para la razón. Una fe de carácter fundamentalista puede parecer una fe fuerte porque se enfrenta a la hoy todopoderosa racionalidad científica. Pero cuando la fe lleva a una actitud defensiva ante la ciencia o, en términos más generales, ante la razón, en realidad lo que puede quedar al descubierto es la debilidad de dicha fe.

Una fe como la cristiana, que constituye una fuente inagotable de conocimiento e inspiración, debería llevar a la convicción de que los resultados científicos no pueden amenazar los contenidos de la revelación. Así lo han asumido grandes pensadores de la historia del cristianismo como, por ejemplo, San Agustín o Santo Tomás. El autor de la Naturaleza y de la Revelación, se suele decir, es el mismo y, consiguientemente, no parece razonable que Dios mismo se contradiga en las verdades que ponen de 
manifiesto sus criaturas, y las que él haya querido revelarnos por otros medios. Considero que no es pertinente que el hombre de fe se encierre en unas formulaciones particulares, y necesariamente limitadas, por estar expresadas en lenguaje humano. Debería ser más bien un continuo buscador, alguien que trata de comprender cada vez mejor aquello que pueda ser verdad provenga de donde provenga, que no ve en la ciencia una amenaza sino una ayuda para comprender mejor lo que sabe sobre Dios, y su relación con Él, por otras vías. Tomás de Aquino se consideraba a sí mismo "prisionero de la verdad". Por esto su gran reto fue comprender las verdaderas aportaciones del conocimiento humano, vinieran de donde vinieran. Su fe le llevaba a tener el convencimiento de que podrían ayudarle a comprender las realidades divinas.

En definitiva, en este contexto, considero que la fe no tiene como misión facilitarnos el camino en el control de la realidad física. Según la fe cristiana expresada en el libro del Génesis, esta es una tarea encomendada al hombre por Dios. Y en esta tarea las diversas ciencias nos están ayudando a cumplir con dicho mandato de una manera cada vez más eficaz. En cambio, la fe nos da a conocer a Dios y nos ayuda a comprender mejor nuestra relación personal con Él y con el mundo que habitamos y dominamos.

Juan Pablo II expresó estas ideas en numerosas ocasiones con especial claridad. Un ejemplo es el discurso pronunciado a la Academia Pontificia de las Ciencias en 1981: "La Biblia nos habla del origen del universo y de su constitución, no para proporcionarnos un tratado científico, sino para precisar las relaciones del hombre con Dios y con el universo. La Sagrada Escritura quiere declarar simplemente que el mundo ha sido creado por Dios, y para enseñar esta verdad se expresa con los términos de la cosmología usual en la época del redactor. El libro sagrado quiere además comunicar a los hombres que el mundo no ha sido creado como sede de los dioses, tal como lo enseñaban otras cosmogonías y cosmologías, sino que ha sido creado al servicio del hombre y para la gloria de Dios. Cualquier otra enseñanza sobre el origen y la constitución del universo es ajena a las intenciones de la Biblia, que no pretende enseñar cómo ha sido hecho el cielo sino cómo se va al cielo. Cualquier hipótesis científica sobre el origen del 
mundo, como la de un átomo primitivo de donde se derivaría el conjunto del universo físico, deja abierto el problema que concierne al comienzo del universo. La ciencia no puede resolver por sí misma semejante cuestión: es preciso aquel saber humano que se eleva por encima de la física y de la astrofísica y que se llama metafísica; es preciso, sobre todo, el saber que viene de la revelación de Dios".

\subsection{Impertinencias contra la religión}

En este contexto y, en términos muy generales, pienso que las impertinencias más importantes contra la religión han sido dos. Ambas parecen situarse en extremos opuestos, pero considero que son más próximas entre sí de lo que pudiera parecer.

Por una parte es impertinente excluir a priori de lo que se considera real a todo aquello que no pueda ser explicado por la ciencia. Si la ciencia no es capaz de explicar algo que llama a las puertas de nuestro conocimiento, sería impertinente afirmar que, o bien lo podrá explicar en el futuro, o bien simplemente afirmar que esa realidad no existe y, por tanto, no supone ningún problema su explicación porque, sencillamente, no hace falta hacerlo.

En este caso se hace un acto de fe en la ciencia y se supone que una racionalidad que nos ha proporcionado tantos beneficios, y que ha conseguido mejores niveles de consenso en sus aportaciones que cualquier otro tipo de racionalidad, podrá explicarlo todo.

La impertinencia se hace especialmente aguda cuando se defiende que es la ciencia dominante, el paradigma imperante, el que tiene el poder de dar respuesta a todos los problemas e interrogantes que podemos plantearnos.

Parece razonable que, desde una perspectiva semejante, o bien se rechaza la religión como producto de una racionalidad trasnochada que supone un obstáculo al progreso del verdadero conocimiento (tesis del conflicto) o, como mucho, se le concede graciosamente una reserva bien acotada para que pueda vivir pacíficamente, sin colisiones ni conflictos, con el único tipo de racionalidad capaz de proponer verdades asumibles por todos (tesis del doble magisterio). Ese reducto es el de la subjetividad. 
Una subjetividad que entonces tiene poco que ver con lo verdadero y que es expresión de una libertad entendida como autoafirmación, pero desconectada de las auténticas verdades.

Por otro lado, también es impertinente la pretensión de servirse de la ciencia para demostrar lo que uno ya ha asumido como verdadero desde la fe: es uno de los casos incluidos en la categoría denominada anteriormente como "integración”. En concreto sería algo así como decir: "la ciencia demuestra la fe". Algunas versiones del creacionismo son ejemplos de esta posición. Pero como las ciencias naturales son especialmente refractarias a cualquier imposición externa, dichas versiones creacionistas han intentado desarrollar una ciencia que evite el conflicto con la propia fe, a la medida de "su fe". La versión del creacionismo que se ha denominado "creacionismo científico", desarrollado especialmente en los años setenta, iría en esta dirección.

Estas dos posiciones son antagónicas. En Estados Unidos lo son también en el terreno político. En un caso la fe es expresión de una subjetividad "libre", y en el otro caso la fe es considerada la fuente prioritaria de conocimiento, incluido el científico. Pienso que ambas tienen una base común que hace posible que sus posiciones sean impertinentes y enfrentadas: reducen o, al menos, otorgan la primacía de lo que significa conocer la verdad a la racionalidad científica.

No deja de ser paradójico, como señala Karl Giberson (Giberson 2011, 205-221), que algunos científicos o divulgadores científicos estén consiguiendo adornar a la ciencia con los atributos que siempre se han considerado propios de la religión. Ellos entonces se convierten en los nuevos teólogos y predicadores, los oráculos, de la nueva religión.

\section{Conclusión}

En el presente estudio he ofrecido una panorámica de las posiciones existentes respecto a la relación entre ciencia y religión. He tratado de poner de manifiesto la complejidad que presenta la sistematización de dichas relaciones y he adoptado un enfoque algo diferente al tratar de delatar 
lo que en esa relación es fuente de inspiración y lo que es impertinente, proceda de donde proceda.

En el momento actual son muy patentes posiciones impertinentes frente a la ciencia y frente a la religión. Los casos extremos son el rechazo de la religión desde un cientificismo puramente materialista y, en el lado opuesto, el rechazo de las ciencias experimentales amparándose en unas creencias que entran en conflicto con ella. Paradójicamente, en estos dos extremos, hemos visto que se podría decir que, o bien se hace de la ciencia una especie de religión, o bien se pretende hacer de la religión una ciencia natural.

En este trabajo se defiende que en ambos casos subyace lo que podríamos llamar una "fe deficiente". O bien porque se deposita una fe sobre un tipo de racionalidad cuyo método tiene la propiedad de excluirla, o bien porque la fe llega a convertirse en un reducto donde protegerse de tesis que parecen contradecir las propias creencias. Lo que podríamos denominar una "fe perezosa".

Las ciencias naturales, en contra de lo que podría pensarse en determinados momentos de la historia, como el siglo XIX respecto a la Física, no están agotando la verdad que podemos encontrar en la realidad. Algunos de sus oráculos actuales así parecen afirmarlo. Es cierto que cada vez conocemos más y mejor los procesos físicos. Pero, curiosamente, este mayor conocimiento nos está revelando la existencia de un mundo material que es más complejo de lo que nosotros habíamos previsto. Una mayor comprensión de la realidad nos permite vislumbrar mejor su enorme complejidad.

Esta situación puede llevar a dos posiciones bien distintas. A una actitud contemplativa y de asombro que es la propia de una aproximación filosófica a la realidad y, consiguientemente, a ampliar el ámbito de la racionalidad. Por otra parte, cuando todas las esperanzas en lo que la racionalidad humana puede dar de sí se depositan en lo que es capaz de ofrecer la ciencia, entonces es fácil caer en el escepticismo. El escepticismo comporta una pérdida de confianza en la razón. Es la consecuencia natural de una esperanza que ha sido defraudada: la ciencia no es capaz de dar razón de aquello que escapa a su propio método. Incluso tratar de 
comprender a la misma ciencia exige ejercerla y, a la vez, trascenderla: hacer filosofía.

En definitiva, pienso que uno de los problemas más importantes que da lugar a las impertinencias mencionadas consiste en la crisis de verdad que hay instalada en la cultura actual. Crisis que fue delatada por Juan Pablo II en la encíclica "Fides et ratio", y que supone un quebranto de confianza en la capacidad de la razón humana para alcanzar auténticas verdades. Ante dicha desconfianza las dos posturas extremas son entregar en exclusiva el conocimiento a la racionalidad científica, que ofrece resultados tangibles y muy útiles; o entregarlo en manos de ideologías, que ofrecen "seguridades”. Si la religión sirviera para dispensarnos del dificultoso trabajo de pensar, entonces sería una ideología más. Entonces el conflicto entre ciencia y religión sería simplemente un conflicto entre ideologías, o bien la consecuencia natural de una cultura dominada por una racionalidad científica que excluye y trata de arrinconar a la religión sin percibir que, entonces, esa "racionalidad científica" se habría convertido ella misma en ideología.

\section{Referencias}

Artigas, M. 1989. “Nicolás Oresme, gran maestre del Colegio de Navarra, y el origen de la ciencia moderna.” Príncipe de Viana (Suplemento de ciencias) 9:297-331.

-. 1999. Filosofía de la Ciencia Experimental. Pamplona: Eunsa.

-. 2000. La mente del Universo. Pamplona: Eunsa.

Carlson, R.F., ed. 2000. Science \& Christianity: Four Views. Illinois: InterVarsity Press. Concilio Vaticano II. 1965. Gaudium et spes, Constitución pastoral sobre la Iglesia en el mundo actual (7-XII-1965).

Ecklund, E.H., and E. Long. 2011. "Scientists and Spirituality." Sociology of Religion 72: 253-74. Accessed june 4, 2013. DOI: http://dx.doi.org/10.1093/socrel/srr003

Ferngren, G.B., ed. 2002. Science \& Religion. A Historical Introduction. London: The Johns Hopkins University Press.

Giberson, K. 2011. “When Science Becomes Religion.” In Science and Faith within Reason. Reality, Creation, Life and Design, edited by J. Navarro, 205-21. England: Ashgate. 
Graffin, G.W., and W.B. Provine. 2008. “Religión y libre albedrío. ¿Qué piensan los biólogos sobre el modo en que se relacionan religión y evolución?” Investigación y Ciencia 377.

Harrison, P. 2010. “'Science' and 'religion': constructing the boundaries.” In Science and Religion. New Historical Perspectives, edited by T. Dixon, G. Cantor, and S. Pumfrey, 23-50. Cambridge: Cambridge University Press.

Jaki, S.L. 2002. A Mind's Matter: An Intellectual Autobiography. Grand Rapids: Eerdmans.

Jaki, S.L.1990. Science and Creation. Washington: University Press of America. Juan Pablo II. 1998. Encíclica Fides et Ratio (14-IX-1998).

Udías, A. 2010. Ciencia y religión. Dos visiones del mundo. Santander: Sal Terrae. Whitehead, A.N. 1967. Science and the Modern World. New York: Macmillan. 